\title{
El síndrome de Chilaiditi en el diagnóstico diferencial del cólico renal
}

\author{
C. Alcober Pérez, E. Carod Benedico, D. Sanz Martínez \\ Médicos Especialistas en Mediana Familiar y Comunitaria. Centro de Salud \\ de Biescas. Huesca
}

\section{RESUMEN}

El síndrome de Chilaiditi es la interposición de una parte del colon entre el hígado y el diafragma. Es un cuadro de muy escasa frecuencia y que res ponde generalmente de forma favorable a trata mientos conservadores. Consideramos de gran in terés conocerlo dado la gran variabilidad de sus formas de presentación clínica, que pueden mime tizar otras entidades. En estos casos, una radio grafía simple de tórax o abdomen puede bastar pa ra el reconocimiento de esta entidad.

Presentamos el caso de un varón joven que pre sentó clínica aguda de intenso dolor lumbar alta mente sugestiva de cólico renal. La realización de pruebas de imagen (radiografía y TAC) hizo posi ble el diagnóstico definitivo.

Palabras clave: Síndrome de Chilaiditi. Cólico renal. Diagnóstico diferencial.
Chilaiditi's syndrome in the differential diagnosis of the renal colic

\begin{abstract}
Chilaiditi's syndrome is the hepato-diaphragma tic interposition of a part of the colon. It's a very low frequent picture and it uses to response favou rably to conservative treatments. We consider im portant to know it since it can have a great variety of clinical presentations, and may mimick other entities. In these cases, simple roentgenograms of the chest or abdomen could be enough to identify this entity.

We report the case of a young man who showed acute symptoms of lumbar pain highly suggestive of renal colic.The study with image tests (radio graphy and computerized tomography) made pos sible the final diagnosis.
\end{abstract}

Key words: Chilaiditi's syndrome. Renal colic. Differential diagnosis.

\section{INTRODUCCIÓN}

El síndrome de Chilaiditi es una alteración anatómica que consiste en la interposición de una parte del colon, generalmente el derecho, entre el hígado y el diafragma. Sin embargo, también ha sido descrita la interposición en el lado izquierdo ${ }^{1}$. De esta infrecuente anomalía posicional existen referencias en la literatura desde el siglo XVIII, aunque no fue hasta 1910 cuando Chilaiditi lo describió en profundidad aportando su nombre a esta entidad nosológica ${ }^{2}$.

La presencia del signo de Chilaiditi, entendiendo como tal el hallazgo casual de la imagen característica en un paciente asintomático ${ }^{3}$, se presenta con una incidencia aproximada de 0,02 a $0,14 \%$ de to- 
das las exploraciones radiológicas toracoabdominales. Es más frecuente en varones mayores de 65 años ${ }^{4}$. Se ha observado una mayor incidencia en los pacientes con cáncer de pulmón (1\% de los casos), así como en ciertos casos de retraso mental ${ }^{5-7}$.

Es mucho más rara la aparición como síndrome, pese a lo cual debe ser tenido en cuenta en el diagnóstico diferencial de otras entidades, especialmente con aquéllas que cursan con elevación diafragmática y aparición de aire en espacio subdiafragmático.

Su importancia, por tanto, radica en un reconocimiento precoz, ya que puede presentarse clínicamente de forma muy semejante a un cólico renal', y no debe confundirse radiológicamente con otros cuadros de extraordinaria gravedad como el neumoperitoneo ${ }^{9-11}$.

\section{CASO CLÍNICO}

Varón de 27 años, sin alergias medicamentosas conocidas ni otros antecedentes patológicos de interés. No fumador.

Acude a la consulta de Atención Primaria por referir dolor lumbar de unas treinta horas de evolución de tipo cólico. La tarde anterior había acudido a urgencias del Centro de Salud por este motivo; tras etiquetarse como lumbalgia mecánica, le fue administrado diclofenaco intramuscular más un relajante muscular con lo que cedió parcialmente unas horas, tras lo cual el dolor volvió a aumentar en intensidad hasta el momento actual.

El paciente, al que se ve francamente afectado por el dolor, cuenta que el mismo se inició el día anterior de forma brusca y tras un acceso de tos, encontrándose previamente bien. Desde entonces el dolor persiste en fosa lumbar derecha y ángulo costolumbar derecho, irradiándose hacia delante en cinturón y escasamente hacia región genital. Lo define como muy intenso, de tipo cólico y sólo ha presentado sensación nauseosa ocasional coincidiendo con el pico máximo de dolor. No sensación febril ni escalofríos. No disnea. No síndrome miccional ni alteraciones del ritmo deposicional.

A la exploración física, el paciente se muestra inquieto e intenta no moverse porque "cualquier movimiento le aumenta el dolor". Normohidratado y coloreado; afebril; tensión arterial 130/80 y frecuencia cardiaca 88 pulsaciones por minuto. Auscultación cardiopulmonar compatible con la normalidad.

Abdomen blando, depresible, discretamente distendido, sin masas palpables; doloroso a la palpación en hipocondrio derecho, siendo claramente más doloroso al desplazar la mano del explorador hacia la parte más externa. Hepatomegalia de 1-2 traveses de dedo. Signo de Murphy dudoso. Peris- taltismo normal. No signos de irritación peritoneal. Percusión renal positiva, refiriendo dolor en la cara anterior del abdomen al realizar la maniobra.

Ante la hipótesis de que se trate de un cólico renal, se realiza en la consulta tira reactiva de orina, que resulta normal.

Dado lo poco claro del cuadro clínico se deriva al medio hospitalario para continuar estudio.

En el servicio de Urgencias se realiza analítica de sangre y orina en la que se aprecia leucocitosis discreta sin desviación izquierda, sin otros signos de interés. En la radiografía simple de tórax (Fig. 1) se aprecia masa intratorácica de contenido aéreo que emerge desde el hemidiafragma derecho.

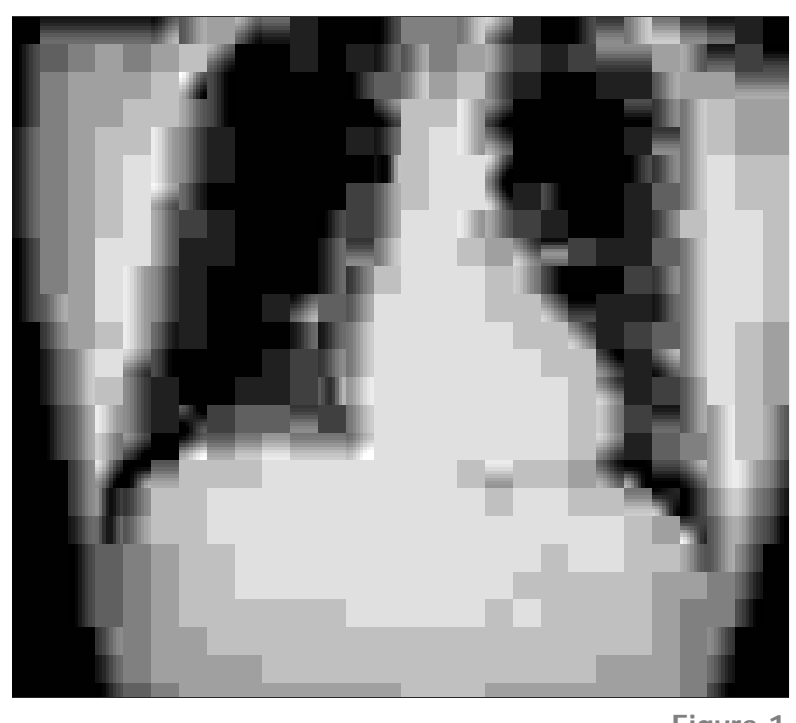

Radiografía de tórax posteroanterior y lateral: imagen aérea característica (signo de Chilaiditi) entre el hemidiafragma derecho y la superficie hepática.

En el TAC toracoabdominal (Fig. 2) se confirma que se trata de intestino grueso que se interpone entre el diafragma y el hígado.

Con el diagnóstico de síndrome de Chilaiditi se inicia tratamiento conservador con analgésicos convencionales cediendo el dolor definitivamente unas 24 horas después.

El paciente refirió después que en la infancia se había realizado alguna exploración radiológica de rutina no habiéndosele informado nunca de la existencia de ninguna alteración.

Desde entonces continúa asintomático.

\section{DISCUSIÓN}

El síndrome de Chilaiditi debe ser incluido en el diagnóstico diferencial del cólico renal, ya que puede presentarse clínicamente de forma muy semejante a éste. 


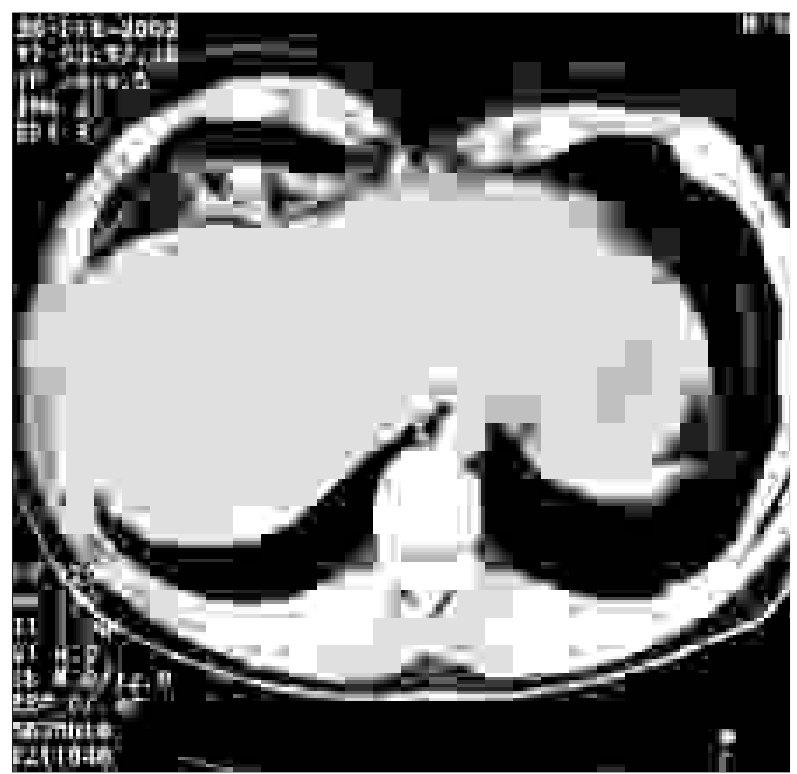

Figura 2

En el TAC se visualiza el colon entre hígado y diafragma derecho confirmándose el diagnóstico de síndrome

de Chilaiditi.

Ha sido relacionado sobre todo con estreñimiento crónico, que para algunos autores se ha postulado como un importante factor predisponente para la interposición colónica ${ }^{12}$. Pese a que la etiología es poco clara, entre los factores predisponentes además del estreñimiento, han sido propuestos otros como: elongación colónica, malposición congénita, parálisis del nervio frénico, ausencia del lóbulo derecho hepático, eventración diafragmática, enfermedad pulmonar obstructiva, adherencias, obstrucción mecánica, cirugía abdominal previa, cirrosis y escoliosis ${ }^{13}$.

Las manifestaciones clínicas más frecuentes son tan inespecíficas como dolor abdominal, vómitos, anorexia y alteraciones del ritmo deposicional (sobre todo estreñimiento). Generalmente son de inicio solapado o progresivo, aunque pueden iniciarse de forma aguda como en el caso que presentamos ${ }^{14,15}$.
En la exploración puede objetivarse distensión abdominal y desaparición de la matidez hepática (signo de Joubert), además de una supuesta hepatomegalia, debida al rechazo del hígado hacia abajo por el colon.

El diagnóstico del síndrome de Chilaiditi es siempre radiológico ${ }^{16,17}$. En una radiografía simple de tórax o abdomen (preferentemente en bipedestación), se apreciará una masa aérea entre el hemidiafragma derecho y la opacidad de la masa hepática. Será identificado el colon de resultar visibles la presencia de haustras. De existir dudas, como en nuestro caso, pueden ser de utilidad el enema con contraste, la gammagrafía, la ecografía o la tomografía axial computarizada ${ }^{18,19}$.

De este modo se hará el diagnóstico diferencial radiológico con el neumoperitoneo, el absceso subfrénico, neumatosis intestinal, quiste hidatídico infectado y tumores hepáticos.

El tratamiento es conservador en la mayor parte de los casos. Consiste en la recomendación al paciente de medidas higiénico-dietéticas, destinadas sobre todo al estreñimiento, a la eliminación de gases y a evitar su formación. Pueden resultar útiles antiespasmódicos, carminativos y laxantes ${ }^{20,21}$. En un episodio agudo con intenso dolor, como nuestro caso, deben administrarse analgésicos, una vez descartadas otras etiologías del dolor.

En los casos complicados con obstrucción intestinal, el tratamiento será quirúrgico.

Creemos fundamental para el médico de familia conocer, y que el propio paciente conozca (dado lo infrecuente de esta patología), que quienes presentan esta interposición colónica, desarrollan vólvulos de colon con mayor frecuencia que la población general $^{22,23}$, por lo que debe pensarse siempre en ello ante un cuadro de dolor agudo en un paciente con dicha anomalía.

\section{CORRESPONDENCIA:}

Carlos Alcober Pérez

C/ Aldebarán, 54, $2^{\circ} \mathrm{D}$

50012 Zaragoza

\section{Bibliografía}

1. Gallego Soriano MJ. Síndrome de Chilaiditi. Revisión a propósito de un caso. Rev Esp Enferm Digest 1983; 63 (1): 66-71.

2. Chilaiditi D. Zur Frage der Hepatoptose und Ptose im allgemeinen im Anschluss an drei Falle von temporarer, partieller Leberverlagerung. Fortcshr Geb Rontgenstr Nuklearmed Erganzongsband 1910-11; 16: 173-208.

3. Plorde JJ, Raker EJ. Transverse colon volvulus and associated Chilaiditi's Syndrome: Case report and literature review. Am J Gastroenterol 1996; 91: 2613-6.

4. Torgerson J. Suprahepatic interporsition of the colon and volvulus of the cecum. Am J Roentgenol 1951; 66: 747-51.

5. Lekkas CN, Lentino W. Symptom-producing interposition of the colon. JAMA 1978; 240: 747-50.

6. López Sendón JL. Cáncer primitivo de pulmón y síndrome de Chilaiditi. Rev Clin Esp 1973; 128: 343-4.

7. López Sendón JL. Primary Lung cancer and the Chilaiditi Syndrome. Chest 1975; 67(1): 130.

8. Ángulo Cuesta J, González Zorraquino A, Unda Urzaiz M, Flores Corral N. Síndrome de Chilaiditi en el diagnóstico diferencial del cólico renal. Arch Esp Urol 1991; 44 (3): $300-1$ 
9. Rodríguez Cuartero A, Peláez Redondo J. Interposición hepato-diafragmática del colon (síndrome de Chilaiditi). A propósito de cuatro observaciones. Rev Esp Enferm Dig 1973; 39: 179-86.

10. Haddad CJ, Lacle J. Chilaiditi's syndrome. A diagnostic challenge. Postgrad Med 1991; 89 (4): 249-52.

11. Walsh SD, Cruikshank JG. Chilaiditi Syndrome. Age Ageing 1977; 6 (1): 51-7.

12. Javors BR, Sorkin NS, Flint GW. Transverse colon volvulus: A case report. Am J Gastroenterol 1986; 81: 708-10.

13. Schubert SR. Chilaiditi's syndrome: an unusual cause of chest or abdominal pain. Geriatrics 1998; 53 (6): 85-8.

14. Risalti A, De Anna D, Terrosu G, Uzzau A, Carcoforo P, Bresadola F. Chilaiditi's syndrome as a surgical and nonsurgical problem. Surg Gynecol Obstet 1993; 176 (1): 55-8.

15. Hisham AN, Gunn A, Jamil AA. Chilaiditi's syndrome presenting as acute abdomen. Med J Malaysia 1995; 50 (3): 281-3.

16. Ebata K, Yamashita H, Yokoyama S. X-ray of Chilaiditi syndrome. Rinsho Hoshasen 1965; 10(8): 607-13.
17. van Everdingen KJ, Feldberg MA. Diagnostic image Chilaiditi syndrome. Ned Tijdschr Geneeskd 2001; 145 (42): 20-6.

18. Changchien CS. Sonographic findings in Chilaiditi syndrome. J Clin Ultrasound 1995; 23 (1): 49-51.

19. Yamamoto S, Fukushima K, Nagao C, Hino K, Ohumi Y, Hirano H. CT appearance of Chilaiditi syndrome. Rinsho Hoshasen 1983; 28 (5): 619-21.

20. Burger L. Clinical aspects and therapy of Chilaiditi Syndrome. Zentralbl Chir 1982; 107 (8): 468-72.

21. Meneses Fernández $\mathbf{M}^{\mathrm{a}} \mathrm{M}, \mathrm{Marchena}$ Gómez J, HernándezSiverio González N, García Santos J, Gómez Culebras M, González Hermoso F. Síndrome de Chilaiditi. Aportación de tres casos. Rev Esp Enferm Dig 1989; 75 (3): 300-3.

22. Orangio GR, Fazio VW, Winkelman E, McGonagle BA. The Chilaiditi syndrome and associated volvulus of the transverse colon: An indication for surgical therapy. Dis Colon Rectum 1986; 29: 653-6.

23. Zinkin LD, Katz LD, Rosin JD. Volvulus of the transverse colon: report of case and review of the literature. Dis Colon Rectum 1979; 22: 492-6. 